\title{
CAMBIOS ECONÓMICOS, COMPETITIVIDAD Y BIENESTAR DE LA POBLACIÓN EN LA REgIÓN NOROESTE de MÉXICO EN LA GLOBALIZACIÓN
}

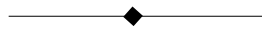

\section{RESUMEN}

La apertura sin precedentes de la economía mexicana al mercado externo, ha traído un conjunto de transformaciones y ha generado mayores expectativas económicas; sin embargo, a 15 años de iniciada, es importante evaluar no solamente los beneficios en términos del aumento en el comercio, sino también los cambios en el bienestar de la población. Para ello, seleccionamos una región: el Noroeste de México (NOR), definida como el espacio geográfico que comprende los estados Baja California, Baja California Sur, Nayarit, Sinaloa y Sonora, que representa $21 \%$ del territorio nacional, $8.7 \%$ de la población, y $9.09 \%$ del producto interno bruto (PIB).

En este trabajo encontramos cambios desde 1988, primero en la estructura económica: PIB, empleo y sector externo. Segundo, encontramos que en la última década hubo un empeoramiento en el bienestar de los hogares. De esta forma, la política de apertura comercial de México hacia el mundo no ha producido, hasta ahora, los beneficios esperados para los hogares del Noroeste de México, región inserta en forma activa en el mundo globalizado, no solamente con Norteamérica, sino también en la cuenca del Pacífico.

\section{ABSTRACT}

The widely straightforward opening of the Mexican economy to the international market, has provided a group of transformations and has generated more economic expectations; however, from a distance of 15 years that the procces has been brought into life, it is important to verify not only the benefits in terms of the trade increasing, but also the changes in the population's well-being. Therefore, we have selected a region: the N orthwestern of M exico (NwR), defined as the geographic space which resembles the states of Baja California, Baja

* CIAD, A.C. Hermosillo. 
California Sur, Nayarit, Sinaloa, and Sonora, with $21 \%$ of the national territory, $8.7 \%$ Mexico's total population, and $9.09 \%$ of the gross domestic product (GDP).

In this paper, we observed changes upon 1988, firstly on the economic structure: GDP, employment, and external sector. Secondly, we found that, for the last decade, the well-being level worsened-off. In this way, the open trading policy from Mexico to the world has not produced, until now, the required expected benefits to the households and the Northwestern region of Mexico, inserted in an active way in the internationalized world, not only with North America, but also with the Pacific Basin region.

\section{INTRODUCCIÓN}

Sin duda, una de las mayores controversias que se han presentado en el ámbito económico y social en la última década en México, se refiere a los beneficios que traería consigo la inserción más intensa de nuestro país en el mundo globalizado, como si con la firma de los tratados comerciales a partir de 1986 como el Acuerdo General de Aranceles y Comercio (GATT), por sus siglas en inglés, organismo que antecedió a la actual Organización Mundial de Comercio (OMC), el Tratado de Libre Comercio (TLC) en 1993, y posteriormente con la firma de tratados con Chile, Centroamérica, y más recientemente con la Comunidad Europea e Israel, se hubiese encontrado la clave para la solución de los problemas que han aquejado a nuestra economía en los últimos 20 años: bajo crecimiento económico, inestabilidad de precios, pobreza y desigualdad.

Es incuestionable que la apertura de la economía mexicana a los mercados ha traído aparejado un conjunto de transformaciones y generado expectativas de mayores cambios que fueron tratadas y cel ebradas, en su momento, por diferentes autores (Bazdresch et al., 1992; Rubli y Solís, 1992; Vega, 1991); sin embargo, a 15 años de iniciado dicho proceso de apertura, es importante revisar no solamente el impacto del nuevo modelo en la economía globalizada (De la Torre R., 2000), sino también los cambios en las regiones de nuestro país, en términos de incremento de los flujos comerciales, del empleo, la competitividad y el bienestar de la población. Para tal propósito, hemos seleccionado una región que ha mantenido, desde antes de la misma liberalización comercial, un activo intercambio con el exterior: la región N oroeste de México (RNO). 
En este trabajo, nuestro objetivo será analizar los cambios observados en la región Noroeste de México en el periodo 1988-1996, tomando como base del análisis los indicadores del PIB, del empleo y del sector exportador de mercancías a partir de los cuales elaboramos los índices de especialización y competitividad. También, tenemos como objetivo conocer los efectos producidos en los niveles de bienestar de los hogares de la región.

Cabe destacar que los años considerados comprenden una etapa de cambios estructurales de la economía mexicana caracterizados por la implementación de un modelo de desarrollo hacia fuera, en contraste con el que había practicado México en los 40 años anteriores. Asimismo, los datos estadísticos disponibles para el periodo nos permiten comparar simultáneamente los cambios en los indicadores económicos y los índices de la participación de la región en el sector externo, así como los cambios en los índices de bienestar, que no eran posibles para años anteriores.

Nuestra hipótesis enuncia que los cambios sufridos por la economía mexicana como efecto de la apertura a los mercados externos, la firma de tratados comerciales y la globalización, no han producido aumento en el bienestar de la población de la región N oroeste, como tampoco en alguna de las entidades que la conforman, pues al parecer fue mayor el impacto negativo de la crisis sufrida por nuestra economía a mitad de la última década del siglo XX, que los beneficios de la globalización en el bienestar de los hogares en la RNO.

\section{LA ESTRUCTURA ECONÓMICA DE LA REGIÓN NOROESTE DE MÉXICO (RNO)}

La RNO, definida como el espacio geográfico que conforman los estados de Baja California, Baja California Sur, Nayarit, Sinaloa y Sonora (Bassols, 1972), ocupa 21\% del territorio mexicano. La dimensión de la región y las entidades respectivas en el contexto nacional, podemos comprobarla con base en indicadores de población y económicos que aparecen en el cuadro 1. En 1996, la RNO contribuía con $8.7 \%$ de la población total del país, y con $9.09 \%$ del PIB; esto es, aportaba un punto porcentual por cada punto de población. El 
PIB per cápita ascendía a 13326 pesos de 1993, ligeramente superior a los 12767 del promedio nacional. Contribuía también con $9.5 \%$ del empleo, y en 1998 con 7.5\% del valor de 110431 millones de dólares de las exportaciones totales.

La economía de la región muestra una diversificación en la que destaca un sector moderno ligado a las exportaciones: a) agrícolas, en las que predominan las hortalizas, ganado, pescados y mariscos (Sinaloa, Sonora y Baja California Sur); b) maquiladoras, con productos tales como maquinaria, aparatos electrónicos, material eléctrico y sus partes (Baja California y Sonora), automóviles (Sonora); y c) un sector de servicios y financiero (Baja Cal ifornia). Esto contrasta con una economía más de tipo tradicional orientada al mercado interno con agroindustrias (Sonora, Sinaloa y Nayarit), pesca (Baja California Sur y Nayarit) y turismo (Baja California Sur y Baja Califor-nia).

CUADRO 1 Población, PIBy empleoen Méxicoy la Región Noroeste, 1996.

\begin{tabular}{|l|c|c|c|c|c|}
\hline Entidades & $\begin{array}{c}\text { Poblacióntotal } \\
\text { (enmiles) }\end{array}$ & PIB $^{1}$ & $\begin{array}{c}\text { PIB/ } \\
\text { hab }\end{array}$ & $\begin{array}{c}\text { Empleo } \\
\text { (enmiles) }\end{array}$ & Exportaciones $^{2}$ \\
\hline EstadosUnidos & 93234 & 1190344 & 12767 & 26924 & 110431 \\
Mexicanos & $(\%)$ & $(\%)$ & & $(\%)$ & $(\%)$ \\
RegiónNoroeste & 8.71 & 9.09 & 13326 & 9.50 & 7.15 \\
BajaCalifornia & 2.37 & 295 & 15865 & 269 & 4.81 \\
B.CaliforniaSur & 0.42 & 0.58 & 17729 & 0.54 & 0.03 \\
Nayarit & 0.98 & 0.61 & 7896 & 1.08 & 0.02 \\
Sinaloa & 2.65 & 2.24 & 10790 & 2.87 & 0.38 \\
Sonora & 2.30 & 272 & 15125 & 233 & 1.92 \\
\hline
\end{tabular}

${ }^{1}$ Millones de pesos de 1993.

2 Millones de dólares de 1998.

Fuente: Estimaciones propias con base en: INEGI, Censos G enerales de Población y Vivienda, 1990 y Conteo de Población, 1995; Cuadernos de Información 0 portuna R egional, 2do. Semestre 1995 y 1996, y Sistema de Cuentas Nacionales, 1999. Bancomext, Exportaciones por entidad federativa, Base de Información, 1998. 
El cómo se ha transformado esta última década influida por la apertura comercial y las crisis, lo analizaremos en seguida.

\section{EL CRECIMIENTO DEL PIB Y EL CRECIMIENTO ECONÓMICO DE LA RNO}

La economía de la región (PIB) creció a una tasa media anual de $2.9 \%$ (véase cuadro 2), por encima de la nacional de $2.6 \%$. La entidad quemostró el mayor crecimiento en el periodo fueBaja California Sur (4.3\%), a diferencia de N ayarit y Sonora, con tasas por debajo de la región e inclusive nacional, de $1.7 \%$ y $2.3 \%$ respectivamente.

Baja California es la entidad de la RNO que aportó un mayor volumen al PIB nacional con $2.95 \%$, seguida por Sonora con $2.72 \%$, no obstante la caída detectada durante el periodo. Sinaloa aportó $2.24 \%$, seguido de Nayarit con $0.61 \%$, y Baja California Sur con apenas $0.58 \%$. Cabe agregar que Sinaloa y Nayarit daban muestras de una economía estancada incluso antes de la crisis de 1995.

CUADRO 2.Productointerno brutodeMéxicoylaregión Noroeste, 1988-1996 (en millonesdepesosde1993). ${ }^{1}$

\begin{tabular}{|l|c|c|c|c|}
\hline Entidades & 1988 & 1993 & 1996 & TMCA 88-93 \\
\hline Estados Unidos & & & & \\
Mexicanos & 966045 & 1155132 & 1190344 & 2.6 \\
Región Noroeste & 8.87 & $(\%)$ & $(\%)$ & \\
Baja California & 2.76 & 2.93 & 9.09 & 2.9 \\
B. California Sur & 0.51 & 0.53 & 2.95 & 3.5 \\
Nayarit & 0.65 & 0.66 & 0.58 & 4.3 \\
Sinaloa & 2.16 & 2.33 & 0.61 & 1.7 \\
Sonora & 2.80 & 2.61 & 2.24 & 3.1 \\
\end{tabular}

1 Índice de precios implícito del PIB, base 1993, La economía mexicana en cifras, varios números, y SCN, Banxico-INEGI, 1999.

FuENTE: Estimaciones propias con base a: Sistema de Cuentas N acionales, Años respectivos, INEGI. 
CUADRO 3. ProductoInternoBrutoper cápitadeMéxicoy laregión Noroeste, 1988-1996(en pesosconstantesde1993). ${ }^{1}$

\begin{tabular}{|l|c|c|c|}
\hline Entidades & $1988^{*}$ & $1993^{*}$ & $1996^{*}$ \\
\hline Estados Unidos Mexicanos & 12363 & 13272 & 12767 \\
Región Noroeste & 13119 & 13846 & 13326 \\
Baja California & 17190 & 16847 & 15865 \\
Baja California Sur & 16751 & 17575 & 17729 \\
Nayarit & 7808 & 8194 & 7896 \\
Sinaloa & 9800 & 11521 & 10790 \\
Sonora & 15396 & 15261 & 15125 \\
\hline
\end{tabular}

1 Índice de precios implícito del PIB.

* Para dichos años setomó como basela población estimada en función de la respectiva TMCA.

Fuente: Estimaciones propias con base en: INEGI, Sistema de Cuentas Nacionales, 1999 y Nafin, La economía mexicana en cifras, 1995.

Aunque la región observó un leve crecimiento, e incluso incrementó su aportación al producto interno bruto a nivel nacional de $8.8 \%$ a $9.1 \%$, en la década de los noventa sufrió una caída en el crecimiento económico (véase cuadro 3), definido éste como el crecimiento del ingreso real per cápita (The World Bank, 2001; Kuznets, 1976), o del crecimiento del PIB per cápita a precios constantes de 1993, como es el caso que estudiamos. La reducción del crecimiento económico coincide con lo ocurrido para México como producto, sin duda, de la crisis de 1994-1995 y que en la RNo afectó en mayor medida a Baja California, Nayarit y Sinaloa. Baja California Sur es la única entidad que logró crecimiento económico, debido más bien al bajo crecimiento de su población que a la dinámica propia de las fuerzas del mercado.

\section{El Producto Interno Bruto Sectorial}

El análisis sectorial revela una disminución de la participación del sector ligado al campo y minero (véase cuadro 4), tendencia observada 
CUADRO 4. Estructuraeconómicasectorial del producto intemobrutodeM éxicoy regiónNoroeste,1988-1996 (en millonesdepesosde1993).

\begin{tabular}{|l|c|c|c|c|c|c|}
\hline \multirow{2}{*}{ Sector económico } & \multicolumn{2}{|c|}{1988} & \multicolumn{2}{c|}{1993} & \multicolumn{2}{c|}{1996} \\
\cline { 2 - 7 } & México & RN O & México & RN O & México & RN O \\
\hline Total & 966045 & 85785 & 1155132 & 103110 & 1190344 & 108185 \\
Agropecuarias, silvicultura y pesca & $(\%)$ & $(\%)$ & $(\%)$ & $(\%)$ & $(\%)$ & $(\%)$ \\
Minería & 7.90 & 16.96 & 6.55 & 13.08 & 6.47 & 12.96 \\
Manufacturas & 2.95 & 2.17 & 1.43 & 1.04 & 1.47 & 1.02 \\
Construcción & 23.86 & 14.07 & 19.23 & 14.10 & 20.28 & 14.95 \\
Electricidad, gas y agua & 3.99 & 3.67 & 4.18 & 3.70 & 4.36 & 3.79 \\
Comercio, hoteles y restaurantes & 1.31 & 1.96 & 1.73 & 2.57 & 1.73 & 2.60 \\
Transporte y comunicaciones & 25.35 & 28.43 & 20.25 & 20.17 & 20.18 & 20.08 \\
Servicios financieros, comunales e importados & 9.46 & 8.94 & 9.82 & 9.64 & 10.08 & 9.79 \\
& 25.18 & 23.79 & 36.80 & 35.71 & 35.42 & 34.81 \\
\hline
\end{tabular}

FuentE: INEGI, Sistema deCuentas Nacionales, producto interno bruto por entidad federativa, 1988 y 1993-1996, M éxico, 1999. 
igual mente para el país desde hace décadas; mientras que la manufactura mantuvo su participación, en la que destaca la sustitución de la industria tradicional por la maquiladora de exportación, particularmente en Baja California.

\section{EL EMPLEO}

El empleo muestra aspectos característicos de la estructura productiva de la región, detectando la importancia en la generación de empleos por actividad económica. La RNO se caracteriza por ser generadora de empleo principalmente en el sector manufacturero, seguido de los servicios y la agricultura, acorde con la tendencia nacional, donde el sector agrícola ha perdido importancia durante el periodo.

En este sentido, el sector agropecuario decreció 2.8\% (véase cuadro 5), que se reflejó en una caída de su participación del $25.2 \%$ al $22.4 \%$ en la estructura ocupacional de la región, y continúa con la tendencia iniciada décadas atrás, que por lo demás coincide con lo observado a nivel nacional con una disminución de 7\%.

La ocupación en la minería muestra una participación estable sin cambios considerables en el periodo.

Por su parte, el sector manufacturero mostró una participación creciente de al rededor de diez puntos porcentuales en la RNO hasta alcanzar $25.78 \%$ en 1996 , un poco por debajo de la aportación nacional en este sector, que fue de un poco más de $28 \%$.

La rama de la construcción disminuyó su participación relativa, tanto en la región como en el país, siendo ésta de $4.8 \%$ y $4.5 \%$, respectivamente, de manera que este sector fue uno de los más afectados por la crisis.

La participación de la ocupación en la electricidad, petróleo, gas y agua en la RNO cayó poco más de un punto porcentual, mientras que para México la caída fue apenas notable (0.23\%). Las ramas de comercio, restaurantes y hoteles, y de comunicaciones y transporte, permanecieron casi inalteradas. En cambio, los servicios aumentaron su contribución en el empleo para la región en 1.75 puntos 
CUADRO 5. Estructuraocupacional por sector económicoen México y la región Noroeste, 1988-1996(miles depersonas).

\begin{tabular}{|c|c|c|c|c|c|c|}
\hline Sector económico & & & 19 & & 199 & \\
\hline & México & RNO & México & RNO & M éxico & RNO \\
\hline Total & $\begin{array}{c}23403.4 \\
(\%)\end{array}$ & $\begin{array}{c}2124.5 \\
(\%)\end{array}$ & $\begin{array}{c}25813.2 \\
(\%)\end{array}$ & $\begin{array}{c}2410.4 \\
(\%)\end{array}$ & $\begin{array}{c}26924.8 \\
(\%)\end{array}$ & $\begin{array}{c}2558.3 \\
(\%)\end{array}$ \\
\hline Agropecuario, silvicultura y pesca ${ }^{1}$ & 22.65 & 25.28 & 15.6 & 21.92 & 15.58 & 22.44 \\
\hline Minería & 0.42 & 0.49 & 0.62 & 0.77 & 0.62 & 0.76 \\
\hline Manufacturas & 19.20 & 15.25 & 28.72 & 24.54 & 28.72 & 25.78 \\
\hline Construcción & 6.82 & 6.79 & 2.33 & 2.13 & 2.33 & 1.98 \\
\hline Electricidad, petróleo, gas y agua & 1.35 & 6.50 & 1.12 & 5.42 & 1.12 & 5.35 \\
\hline Comercio, restaurantes y hoteles & 16.56 & 13.47 & 16.78 & 14.29 & 16.78 & 13.75 \\
\hline Transporte y comunicaciones & 4.47 & 4.25 & 4.83 & 4.41 & 4.83 & 4.25 \\
\hline Servicios financieros, comunales, personales e imputados & 21.14 & 21.17 & 26.59 & 23.59 & 26.59 & 22.91 \\
\hline Admón. pública y defensa ${ }^{2}$ & 3.97 & 4.13 & - & - & - & - \\
\hline No especificados ${ }^{3}$ & 3.43 & 3.55 & 3.43 & 3.56 & 3.43 & 3.56 \\
\hline
\end{tabular}

${ }^{1}$ Para 1995 y 1996 se agregó la diferencia descontado el porcentaje correspondiente a los no especificados del empleo obtenido con el coeficiente de expansión, que corresponde a otros grupos de los Cuadernos de Información Oportuna Regional, núm.44, 20. trim., Cuadro 3.17, p.70, que ayuda a compensar los millones de trabajadores del campo que no aparecen en los asegurados del IMSS, base para estimar el coeficiente de expansión (CE)=Población ocupada censal / asegurados permanentes 1990.

${ }^{2}$ Administración Pública y Defensa se encuentran incluidos en servicios financieros y comunales en 1995 y 1996.

${ }^{3}$ Se mantiene la participación (\%) observada en 1990 para los años 1995 y 1996.

FUENTE: Censos generales depoblación y vivienda, 1980 y 1990; Cuaderno del nformación Oportuna Regional (CIOR) núms.44y 48, 2o. trimestre, 1995 y 1996, INEGI. 
porcentuales para ubicarse en $22.9 \%$, por debajo de la participación relativa de los servicios en México, de $25.6 \%$.

Al interior de la RNO, Sinaloa y Nayarit son las entidades que participan con la mayor proporción de ocupados en el sector agrícola con $29 \%$ y $44 \%$ respectivamente, siguiendo en orden de importancia los servicios con $27 \%$ y $21.5 \%$, y el comercio, restaurantes y hoteles con $20.37 \%$ y $13 \%$.

Baja California Sur y Sonora también tienen un aporte considerable al empleo agrícola de la región con $29.4 \%$ del total regional. Mientras que la entidad que tiene una participación más elevada dentro de los servicios es Baja California Sur, con 33\% de su empleo y un aporte a nivel regional de $8.1 \%$.

Por su parte, Baja California es la entidad con mayor importancia como generadora de empleos en el sector manufacturero con $44 \%$ dentro de su economía, y contribuye con $52 \%$ del empleo manufacturero de la RNO. El caso opuesto lo constituye Nayarit, en donde escasamente participa el sector con $8.8 \%$ de su ocupación y $3.8 \%$ en la región.

En suma, podemos afirmar que tanto el sector manufacturero, el de servicios y el agrícola, son los que generaron la mayor parte del empleo en la región con un total de 1819685 ocupados que representaron el $71 \%$ en 1996, sin dejar de lado la reducción relativa en el empleo del propio sector agrícola en la RNO.

\section{EL SECTOR EXTERnO}

El sector que directamente esperaba beneficiarse con la apertura comercial era el de las exportaciones de mercancías. Para evaluar su desempeño utilizaremos un conjunto de indicadores que ayudan a medir su participación en la economía, así como la intensidad de su desarrollo y su eficiencia durante el periodo.

En lo que se refiere a la participación en las exportaciones, la RNO contribuyó en 1998 con cerca de 8 mil millones de dólares estadounidenses (véase cuadro 6), que representaron $7.15 \%$ del total. En este rubro destaca Baja California con dos terceras partes del valor total de la región. Las mercancías que más se exportaron 
correspondieron a productos de la maquiladora como maquinaria, aparatos eléctricos y electrónicos; reactores y calderas; navegación aérea y espacial; instrumentos y aparatos de óptica; y vehículos, que en su conjunto representaron cerca del $60 \%$ del total. Otras exportaciones fueron: frutas y legumbres, con $5.72 \%$; cobre y sus manufacturas, con $2.63 \%$; y otros productos que se exportaron en proporciones menores a $2 \%$ (véase cuadro 7 ).

Un análisis más detallado acerca del papel del sector exportador en el nuevo contexto económico nos lo proporciona una serie de índices (CEPAL-ONUDI, 1991) que a continuación detallamos.

Primero, el índice de especialización, que representa la importancia que tiene un producto para la región y el país. En la medida en que el índice adquiere valores superiores a la unidad, expresa un mayor nivel de especialización, y viceversa. El índice de especialización (IE) se estima de la siguiente manera:

$$
I E=x_{i j} / x_{j} / \Sigma^{k} X_{j} / \Sigma_{i j}^{n} \Sigma^{k} X_{j}{ }_{i j}
$$

donde:

$\mathrm{x}_{\mathrm{i}}$ : valor de las exportaciones del bien i de la región o estado j

CUADRO 6. Participación delaregión Noroesteen el total deexportaciones deMéxico,1996-1998*(enmillonesdedólaresestadounidenses).

\begin{tabular}{|l|r|r|r|r|}
\hline & 1996 & \multicolumn{1}{c|}{$\%$} & \multicolumn{1}{c|}{1998} & \multicolumn{1}{c|}{$\%$} \\
\hline México & 96000 & 100 & 110431 & 100 \\
Total entidades & 45481.3 & 47.38 & 58054.8 & 52.57 \\
RNO & 6632 & 6.91 & 7896 & 7.15 \\
Baja California & 4345 & 4.53 & 5311 & 4.81 \\
Baja California Sur & 42 & 0.04 & 31 & 0.03 \\
Nayarit & 12 & 0.01 & 17 & 0.02 \\
Sinaloa & 468 & 0.49 & 421 & 0.38 \\
Sonora & 1764 & 1.84 & 2116 & 1.92 \\
\hline
\end{tabular}

FUENTE: CIAD, Estimaciones propias con baseen: Información económica de Bancomext. 
CUADRO 7. Espedialización, posicionamientoy competitividad delaregión Noroeste, 1996-1998.

\begin{tabular}{|c|c|c|c|c|c|c|c|c|}
\hline \multirow[t]{2}{*}{$\begin{array}{c}\text { Fracción } \\
\text { arancelaria }\end{array}$} & \multirow[t]{2}{*}{ Productos $^{1}$} & \multirow{2}{*}{\begin{tabular}{|c|}
$\begin{array}{l}\text { Participación } \\
\text { del producto }\end{array}$ \\
1998 \\
\end{tabular}} & \multicolumn{2}{|c|}{$\begin{array}{c}\text { Índice de } \\
\text { especialización }\end{array}$} & \multirow{2}{*}{$\begin{array}{c}\begin{array}{c}\text { Posiciona- } \\
\text { miento }\end{array} \\
1996 / 98\end{array}$} & \multicolumn{2}{|c|}{$\begin{array}{l}\text { Competi- } \\
\text { tividad }\end{array}$} & \multirow[t]{2}{*}{ Cambio } \\
\hline & & & 1996 & 1998 & & 1996 & 1998 & \\
\hline 85 & $\begin{array}{l}\text { Máquinas, aparatos, material } \\
\text { eléctrico y sus partes }\end{array}$ & 37.57 & 1.52 & 1.57 & $\mathrm{~F}$ & 22.68 & 19.83 & Ваја \\
\hline 84 & $\begin{array}{l}\text { Reactores nucleares, calderas, } \\
\text { maquinaria y aparatos }\end{array}$ & 10.57 & 1.07 & 0.98 & D & 16.50 & 11.38 & Baja \\
\hline 88 & Navegación aérea o espacial & 8.15 & 0.04 & 6.74 & $\mathrm{~F}$ & 3.68 & 92.56 & Alta \\
\hline 7 & $\begin{array}{l}\text { Legumbres y hortalizas, plantas, } \\
\text { raíces y tubérculos }\end{array}$ & 5.72 & 3.37 & 3.03 & $\mathrm{D}$ & 42.76 & 33.51 & Baja \\
\hline 90 & $\begin{array}{l}\text { Instrumentos y aparatos de } \\
\text { óptica, fotografía o cine }\end{array}$ & 3.41 & 1.77 & 1.38 & D & 28.50 & 16.37 & Baja \\
\hline 74 & Cobre y sus manufacturas & 2.63 & 3.03 & 4.38 & $\mathrm{~F}$ & 30.99 & 52.54 & Alta \\
\hline 94 & $\begin{array}{l}\text { Muebles; mobiliario } \\
\text { médico-quirúrgico artículos }\end{array}$ & 2.58 & 1.45 & 1.46 & D & 26.91 & 18.01 & Baja \\
\hline 87 & $\begin{array}{l}\text { Vehículos automotrices, } \\
\text { tractores, ciclos y demás }\end{array}$ & 1.30 & 0.08 & 0.07 & I & 1.1 & 1.1 & Igual \\
\hline 3 & Pescados y crust., molus. y otros invert. & 0.62 & 1.39 & 1.64 & I & 16.73 & 19.53 & Alta \\
\hline 8 & Frutos, cortezas de agrios o de melones & 0.28 & 0.61 & 0.32 & D & 7.55 & 4.06 & Baja \\
\hline
\end{tabular}

${ }^{1}$ Productos ordenados por importancia en las exportaciones de la región en 1998.

$\mathrm{F}=$ Favorable; $\mathrm{D}=$ = Desfavorable; I = Igual.

FuENTE: Estimaciones propias con base en: Bancomext, 1999 ; CEPA L-ONUDI, 1991; y Wong, 1997. 
$\mathrm{x}_{\mathrm{i}}$ : valor de las exportaciones totales de la región o estado j

$X_{i}$ : valor total nacional de las exportaciones del bien $i$

$\mathrm{X}_{\mathrm{j}}$ : valor total nacional de las exportaciones del bien $\mathrm{i}$

En segundo lugar consideramos el posicionamiento, que califica el cambio en la participación de un producto en el mercado, en este caso de las exportaciones, calificándolo de favorable si aumenta y desfavorable si disminuye.

En tercer lugar tomamos el concepto de competitividad que representa la intensidad con que un producto de la región participa el valor total de las exportaciones. El índice de competitividad se obtiene de la siguiente manera (con las variables definidas como se hizo anteriormente):

$$
I C=x_{i j} / \sum_{j}^{k} X_{i j} / x_{j} / \sum_{i}^{n} \Sigma_{j}^{k} X_{i j}
$$

Por último, incluimos el concepto de cambio para calificar los movimientos en la competitividad.

Al tomar dos años de referencia (1996 y 1998), por ser los únicos disponibles que pueden ser comparables para toda la década de los noventa, encontramos lo siguiente. 1) podemos calificar a la RNO como especializada en exportaciones de productos de empresas maquiladoras y de hortalizas, con posicionamiento favorable para la maquila, aunque desfavorable para las hortalizas. Respecto a la competitividad, es interesante observar la disminución de las dos principales ramas de exportación de la región ya señaladas.

En general, basados en los indicadores del comercio exterior de la región, no podemos desprender todavía de manera clara que la RNo haya sido favorecida por la globalización, pues ha ganado en algunas ramas, pero aún en la actividad exportadora más importante, la maquiladora, muestra reducción en la competitividad (véase cuadro 7).

Tal vez las evidencias mostradas hasta aquí no sean suficientes para establecer una conclusión de alcances mayores, sin embargo, nos da una muestra de que las actividades tradicional mente exportadoras de la región, las agropecuarias, están siendo desplazadas. 


\section{EL BIENESTAR Y LA POBREZA}

¿Qué ocurrió con el bienestar de la población de la región RNO en la década de la apertura comercial? Para contestar esta pregunta es necesario definir el bienestar en términos de un concepto que lo haga mesurable. El Banco Mundial, institución reconocida en el tema, ha considerado el ingreso per cápita (Aturupa, Glewwe e Isenman-Banco M undial, 1994) como indicador del bienestar, de tal suerte que los países con ingresos más al tos se dice tienen mayores niveles de bienestar, sin dejar claro cuál es el bienestar al que deben aspirar los individuos, de tal suerte que las políticas de bienestar social se reducen estrictamente a la búsqueda del incremento del ingreso per cápita. Sin embargo, en los últimos 20 años se ha adoptado en la mayoría de los países, con el propósito de implementar programas de combate a la pobreza, una norma que considera el consumo de bienes y servicios que proporciona el mínimo de bienestar para el individuo y el hogar, como el indicador del bienestar.

El mínimo de bienestar considera un conjunto de satisfactores para cubrir las necesidades definidas como básicas, entre las que se encuentran: alimentación, vivienda y sus servicio, educación, salud, transporte, ropa y calzado, cuidado personal y de la casa, muebles y enseres, y cultura y recreación.

El precio de mercado de los satisfactores que proporcionan el mínimo de bienestar para desarrollar las capacidades básicas (Nussbaun y Sen, 1996) se denomina línea de pobreza o costo del mínimo de bienestar. Esta línea divide a la población y hogares entre aquéllos cuyo ingreso les permite obtener el bienestar mínimo y los que, por el contrario, se encuentran en pobreza. Una medida del bienestar $\left(\mathrm{I}_{\mathrm{b}}\right)$ aceptable sería aquélla que nos permitiera saber qué tanto pueden adquirir en el mercado los individuos y los hogares, con un ingreso determinado $\left(Y_{i}\right)$ de ese bienestar mínimo, convertido en una o varias canastas de bienes y servicios que tienen un costo (z) Ilamado línea de pobreza:

$$
\mathrm{I}_{\mathrm{b}}=\mathrm{Y}_{\mathrm{i}} /
$$


De esta manera tenemos:

si $\mathrm{I}_{\mathrm{b}}=1$ el individuo tiene el ingreso suficiente para alcanzar el bienestar mínimo.

si $\mathrm{I}_{\mathrm{b}}<1$ el individuo carece del ingreso suficiente; por lo tanto cae en pobreza.

Ésta es una medida del bienestar derivada del método de las líneas de pobreza de Sen (1976).

Para determinar los bienes y servicios que contienen mínimos de bienestar, utilizamos los criterios de la Canasta Normativa de Satisfactores Esenciales y del Banco Mundial-PNUD, cuyo costo es actualizado con los índices de precios al consumidor por rubro de gasto (Banco de México-INEGI).

A simismo, utilizamos los datos del ingreso por tamaño del hogar provenientes de los Tabulados Temáticos del Censo general de población y vivienda. En virtud de que no existen datos del ingreso de los hogares desagregad os y representativos para las entidades, excepto los publicados en 1990, y debido a que son indispensables para medir los niveles de pobreza utilizando el método de LP, para estimarlo en un año diferente al censal consideramos dos escenarios que suponen la evolución del ingreso: 1) el ingreso de los hogares se incrementa conforme lo hace el salario mínimo general de cada entidad; 2) el ingreso se incrementa conforme lo hace el índice general de precios al consumidor por rubro de gasto.

Después, consideramos un modelo de simulación para estimar la pobreza en el que incluimos las variables ingreso (convertida en unidades de adulto equivalente) y precios (Camberos y Huesca, 1997), que juntas nos explican el deterioro de la capacidad de consumo de los hogares, causa fundamental del crecimiento de la pobreza.

Con el propósito de dimensionar mejor los resultados que arroja nuestro trabajo para la región N oroeste y México, los compararemos con los obtenidos en estudios que estiman la pobreza para 1996: Aguilar (2000), Boltvinik y Hernández Laos (1999); Informe Anual del Banco Mundial (2000) y para la región Frontera N orte de M éxico (Camberos y Huesca, 2001). 


\section{EL BIENESTAR AL INICIO DEL PERIODO}

En el cuadro 8A aparece la situación de la pobreza extrema en las entidades de la RNO de acuerdo con el criterio de la CNSE y aplicando el concepto de ingreso por adulto equivalente, para hacer más precisas las estimaciones de la pobreza (Smeeding, 1990). Observamos que para 1990 la pobreza extrema afectaba a 39.3\% de los hogares que habitaban la región Noroeste, 14 puntos porcentuales inferior al índice nacional, que fue de $53.4 \%$. El estado de Nayarit, con $66.9 \%$ de los hogares, aparecía con el índice de pobreza extrema más elevado (48.8\%); en un nivel intermedio estaban los estados de Sinaloa (42.8\%), Sonora (41.0\%) y Baja California Sur (38.2\%). Destaca el estado de Baja California con $29.1 \%$ de los hogares en situación de pobreza extrema.

\section{LA EVOLUCIÓN DEL BIENESTAR EN LOS HOGARES DEL NOROESTE DE MÉXICO BAJO DOS ESCENARIOS, 1990-1996}

De acuerdo con los escenarios planteados, encontramos que en el escenario 1 (cuadro 8A), cuando el ingreso de los hogares se incrementa conforme lo hace el salario mínimo general de cada entidad, en el año 1996 se acentúa la situación de pobreza extrema en la RNO, alcanzando al $55.5 \%$ de los hogares, en tanto que a nivel nacional el $62.1 \%$ estaba en dicha situación.

En lo referente a las entidades de la región, encontramos la mayor incidencia de pobreza extrema en Nayarit (69.8\%) y Sinaloa (64\%), superando ambos estados el índice de pobreza extrema de la región y el país. En nivel intermedio estaban Sonora (58.0\%) y Baja California Sur (56.0\%). El estado de Baja California, con $46 \%$ de los hogares, aparecía con la menor incidencia (44.3\%).

En el escenario 2, cuando el ingreso se incrementa conforme lo hace el índice general de precios al consumidor por rubro de gasto, la RNO pudo haber observado en 1996 39.3\% de las familias en pobreza extrema, y el país 53.4\%, igual que en 1990.

Los resultados para México con el escenario 1, de $62 \%$, son congruentes con los obtenidos por Boltvinik (1999:5): 66 millones de 
pobres extremos con la LPE de CEPAL-INEGI, equivalente a cerca de $68 \%$ de la población.

Al comparar los resultados entre regiones, encontramos que en la frontera norte de México (FNM), que comprende los estados de Baja California, Chihuahua, Coahuila, Nuevo León, Sonora y Tamaulipas, la pobreza extrema al canzó a casi 59\% de la población (Camberos y Huesca, 1997), tres puntos porcentuales menos que la RNO. Lo anterior significa que el ingreso de los más pobres de los pobres, incluso de la FNM, considerada la región más moderna y ligada al sector externo de México, se deterioró realmente en el periodo 1990-1996, lo que provocó el aumento de la pobreza extrema.

Respecto a la evolución de la pobreza que incluye la pobreza extrema y la pobreza moderada, en el cuadro 8B se puede observar que en 1990 la región N oroeste tenía 58\% de los hogares en pobreza,

CUADRO 8A. Estimaciones dePobrezaExtremacon el criterio deCoplamar y el concepto deingreso por adulto equivalentebajo dos escenarios en laregión Noroeste, 1990-1996.

\begin{tabular}{|l|c|c|c|}
\hline Entidades & $\begin{array}{c}\text { Pobreza Extrema } \\
1990\end{array}$ & $\begin{array}{c}\text { Escenario 1: } \\
1996\end{array}$ & $\begin{array}{c}\text { Escenario 2:2 } \\
1996 \\
(\%)\end{array}$ \\
\hline M éxico & 53.4 & 62.1 & 53.4 \\
Región Noroeste & 39.3 & 55.5 & 39.3 \\
Baja California & 29.1 & 44.3 & 30.5 \\
Baja California Sur & 38.2 & 56.0 & 40.2 \\
Sonora & 41.0 & 58.0 & 41.0 \\
Sinaloa & 42.8 & 64.8 & 47.7 \\
Nayarit & 48.8 & 69.8 & 53.8 \\
\hline
\end{tabular}

${ }^{1} \mathrm{El}$ incremento del ingreso es de acuerdo con el aumento salarial.

${ }^{1}$ El incremento del ingreso delos grupos es igual al del salario mínimo por zona: A: Baja California y Baja California sur; B: Sonora y México; C: Sinaloa y Nayarit.

${ }^{2}$ El incremento del ingreso es igual al incremento en precios de las mercancías que constituyen las líneas de pobreza.

FuENTE: Centro deInvestigación en Alimentación y Desarrollo (CIAD); Mario Camberos Castro, Crisis, ajuste económico y pobreza regional. 
CUADRO 8B. Estimaciones depobrezacon el criterio deCoplamar y el concepto deingreso adul to equivalentebajo dosescenarios en la región Noroeste, 1990-1996.

\begin{tabular}{|l|c|c|c|}
\hline Entidades & $\begin{array}{c}\text { Pobreza* } \\
1990\end{array}$ & $\begin{array}{c}\text { Escenario } 1:^{1} \\
1996\end{array}$ & $\begin{array}{c}\text { Escenario } 2:^{2} \\
1996 \\
(\%)\end{array}$ \\
\hline México & 69.7 & 79.1 & 72.2 \\
Región Noroeste & 58.0 & 72.5 & 61.3 \\
Baja California & 46.6 & 61.8 & 45.5 \\
Baja California Sur & 58.5 & 73.0 & 57.3 \\
Sonora & 60.6 & 77.3 & 63.9 \\
Sinaloa & 61.8 & 77.2 & 68.4 \\
Nayarit & 66.9 & 80.6 & 71.9 \\
\hline
\end{tabular}

* La pobreza incluye pobreza moderada y pobreza extrema.

${ }^{1}$ El incremento del ingreso delos grupos es igual al del salario mínimo por zona: A: Baja California y Baja California sur; B: Sonora y México; C: Sinaloa y Nayarit.

${ }^{2}$ El incremento del ingreso es igual al incremento en precios de las mercancías que constituyen las líneas de pobreza.

FuENTE: Centro del nvestigación en Alimentación y Desarrollo (CIAD); M ario Camberos Castro: Crisis, ajuste económico y pobreza regional.

mientras que el índice nacional fue de $69.7 \%$. En cuanto a las entidades que conforman la región, encontramos la mayor incidencia de pobreza en Nayarit, con $66.9 \%$ de los hogares. En un nivel intermedio estaban los estados de Sinaloa (61.8\%), Sonora (60.6\%) y Baja California Sur (58.5\%). La menor incidencia la encontramos en Baja California, con el $46 \%$ de los hogares en pobreza.

La evolución de la pobreza en la década de los noventa la podemos observar en el escenario 1 del cuadro 8B. Éste nos muestra que para 1996 la pobreza alcanzó a 72.5\% de los hogares en la región Noroeste y a $79.1 \%$ en el país. En los estados, la mayor incidencia de pobreza fue para Nayarit, con $80.6 \%$; Sinaloa con $77.2 \%$, y Sonora con $77.3 \%$. En nivel intermedio estaba Baja Cal ifornia Sur con $73.0 \%$. En Baja California se presentó la menor incidencia, con 61.8\% de los hogares en pobreza. 
En el escenario 2 se puede observar que en 1996 la RNo registraba 61.3.\% de las familias en pobreza, mientras en el país era $72.2 \%$. Los estados de Nayarit y Sinaloa tenían la mayor incidencia con $71.9 \%$ y $68.4 \%$ de los hogares, respectivamente. En nivel intermedio estaban Sonora con 63.9\% y Baja California Sur con 57.3\%. La menor incidencia se aprecia en Baja California, con $45.5 \%$ de hogares en pobreza.

Al comparar los resultados con los niveles de pobreza estimados por Boltvinik y Hernández Laos (1999) de 75\% para México, y que son similares a los estimados por Aguilar (2000), encontramos que corresponde a un valor entre los escenarios que planteamos, por lo tanto, es también congruente con nuestros hallazgos.

En cuanto a la comparación regional, la FNM observó 75\% (Camberos y Huesca, 1997), casi tres puntos porcentuales por encima del registrado en el Noroeste.

Con el criterio del PNUD, de un dólar per cápita por día para la pobreza extrema y dos dólares para la pobreza moderada, encontramos que en 1990 todas las estimaciones son menores a las anteriormente anotadas (véase cuadro 9A). Así, la pobreza extrema alcanzaba a $16.7 \%$ de las familias en la región, diez puntos porcentuales menor al índice nacional, que se ubicaba en $26.8 \%$. El estado de Nayarit, con $23.9 \%$ de los hogares, y Sinaloa con $19.0 \%$, tenían el mayor porcentaje de pobreza extrema. En nivel intermedio estaban Sonora con $15.9 \%$ y Baja California Sur con $13.6 \%$. El estado de Baja California, con $12.1 \%$, tenía menos pobreza extrema.

En cuanto a la evolución de la pobreza extrema en la última década, y de acuerdo con el escenario 1, encontramos que en 1996 ésta al canzó a $22.8 \%$ de los hogares en la región N oroeste y a $35.0 \%$ en el país. En las entidades de la región, la mayor incidencia fue para Nayarit con $32.7 \%$ y Sinaloa con $26.3 \%$ de los hogares. En nivel intermedio estaban Sonora (22.8\%) y Baja California Sur (20.1\%). La menor incidencia fue para Baja California, con $17.5 \%$ de las familias.

En el escenario 2, para 1996 la RNo presentaba a $16.7 \%$ de los hogares en pobreza extrema y a México con 26.8\%; es decir, la misma incidencia que encontramos en 1990. 
CUADRO 9A. Estimaciones depobreza extrema con el criterio del PNUD

y el concepto deingreso por adulto equivalentebajo dosescenarios paralaregión Noroeste, 1990-1996.

\begin{tabular}{|c|c|c|c|}
\hline Entidades & $\begin{array}{c}\text { Pobreza extrema } \\
1990 \\
(\%) \\
\end{array}$ & $\begin{array}{c}\text { Escenario } 1:^{1} \\
1996 \\
(\%) \\
\end{array}$ & $\begin{array}{c}\text { Escenario } 2:^{2} \\
1996 \\
(\%) \\
\end{array}$ \\
\hline México & 26.8 & 35.0 & 26.8 \\
\hline Región Noroeste & 16.7 & 22.8 & 16.7 \\
\hline Baja California & 12.1 & 17.5 & 11.8 \\
\hline Baja California Sur & 13.6 & 20.1 & 13.0 \\
\hline Sonora & 15.9 & 22.8 & 15.2 \\
\hline Sinaloa & 19.0 & 26.3 & 18.4 \\
\hline Nayarit & 23.9 & 32.7 & 23.3 \\
\hline
\end{tabular}

${ }^{1}$ La pobreza incluye pobreza moderada y pobreza extrema.

${ }^{1}$ El incremento del ingreso delos grupos es igual al del salario mínimo por zona: A: Baja California y Baja California sur; B: Sonora y México; C: Sinaloa y Nayarit.

${ }^{2}$ El incremento del ingreso es igual al incremento en precios de las mercancías que constituyen las líneas de pobreza.

FuENTE. Centro del nvestigación en Alimentación y Desarrollo (CIAD); Mario Camberos Castro, Crisis, ajuste económico y pobreza regional.

En cuanto a los estados, N ayarit observó la mayor incidencia con 23.3\%. En el nivel intermedio estaban Sinaloa (18.4\%) y Sonora (15.2\%). Los estados de Baja California Sur y Baja California tenían el menor porcentaje dehogares en pobreza extrema, con 13\% y $15.2 \%$, respectivamente.

Por otra parte, en el cuadro 9B se aprecia que en 1990 el 36.3\% de los hogares en la región Noroeste estaba en pobreza, en tanto el índice nacional fue de $50.6 \%$. En cuanto a las entidades de la región, se observó la mayor incidencia en N ayarit con $45.6 \%$ y Sinaloa con $39.6 \%$ de los hogares en pobreza. En nivel intermedio estaban Sonora con $37.9 \%$ y Baja California Sur con $35.0 \%$. La menor incidencia la encontramos en Baja California, con $26.6 \%$ de hogares en pobreza. 
En el escenario 1 se percibe que para 1996 la pobreza alcanzó a $43.0 \%$ de las familias en la región N oroeste y a $55.1 \%$ en el país. Por entidades de la región, la mayor incidencia fue para Baja California Sur con $40.0 \%$ y N ayarit con $53.2 \%$. Los estados de Sinaloa con $47.3 \%$, y Sonora con el $43.0 \%$, estaban en un nivel intermedio de pobreza. Baja California, con $30.7 \%$ de los hogares, presentó la menor incidencia de pobreza.

En el escenario 2 se puede observar que para 1996 la región Noroeste tenía 33.3.\% de las familias en pobreza, mientras en el país $47.1 \%$ de los hogares estaba en esta situación. En los estados, Nayarit tuvo la mayor incidencia con $40.0 \%$. En nivel intermedio estaban Sinaloa (33.7\%), Sonora (31.2\%) y Baja California Sur (28.5\%). En Baja Cal ifornia se tuvo la menor incidencia con $22.4 \%$ de hogares en pobreza.

CUADRO 9B. Estimaciones dePobreza con el criterio del PNUD y el concepto de ingreso por adulto equival entebajo dos escenarios, parala región Noroeste, 1990-1996.

\begin{tabular}{|l|c|c|c|}
\hline Entidades & $\begin{array}{c}\text { Pobreza* } \\
1990\end{array}$ & $\begin{array}{c}\text { Escenario } 1:^{1} \\
1996\end{array}$ & $\begin{array}{c}\text { Escenario } 2:^{2} \\
1996 \\
(\%)\end{array}$ \\
\hline México & 50.6 & 55.1 & 47.1 \\
Región Noroeste & 36.3 & 43.0 & 33.3 \\
Baja California & 26.6 & 30.7 & 22.4 \\
Baja California Sur & 35.0 & 40.0 & 28.5 \\
Sonora & 37.9 & 43.0 & 31.2 \\
Sinaloa & 39.6 & 47.3 & 33.7 \\
Nayarit & 45.6 & 53.2 & 40.0 \\
\hline
\end{tabular}

* La pobreza incluye pobreza moderada y pobreza extrema.

${ }^{1}$ El incremento del ingreso delos grupos es igual al del salario mínimo por zona: A: Baja California y Baja California sur; B: Sonora y México; C: Sinaloa y Nayarit.

${ }^{2}$ El incremento del ingreso es igual al incremento en precios delas mercancías que constituyen las líneas de pobreza.

Fuente: CIAD; Mario Camberos Castro, Crisis, ajuste económico y pobreza regional. 
Los resultados para México de $26.8 \%$ de pobreza extrema, son similares a los 26 millones de pobres extremos que aceptaba Sedesol existían en México en 1996, ${ }^{1}$ estimados con base en la línea de pobreza extrema del PNUD-Banco Mundial.

Respecto a la pobreza, que incluye a la pobreza extrema, el Banco Mundial, organismo reconocido en estos trabajos, en su informe más reciente ${ }^{2}$ estimó que $56 \%$ de la población en México en 1996, era deal guna manera pobre, porcentaje muy similar al obtenido en nuestro escenario 1, (55.2\%) lo cual confirma lo adecuado de nuestras estimaciones que anticipamos con el modelo de simulación.

\section{CONCLUSIONES}

Los cambios sufridos en la economía de México y la RNo en la última década, son evidentes, ello derivado de las crisis y los ajustes económicos y la globalización de la década de los noventa del siglo $X X$. Sin embargo, los beneficios esperados para la RNO, no parecen ser muy claros. Por una parte, aunque las dos principales ramas de exportación, la maquiladora y hortalizas, aumentaron su participación en el total de la región, la maquiladora continúa sin integrar cadenas productivas con la industria y servicios nacionales $y$, además, ambas perdieron competitividad nacional.

Por otra parte, las estimaciones sobre la pobreza muestran que hasta $61 \%$ de los hogares en la RNO, no alcanzaba los mínimos de bienestar, que si bien es cierto es un porcentaje menor al promedio nacional, puedeser indicativo dequela apertura comercial deM éxico al mundo no ha producido, hasta ahora, los beneficios esperados para los hogares de la RNO de México, activamente inserta en el mundo globalizado, tanto con A mérica del Norte como en la cuenca del Pacífico.

${ }^{1}$ Declaraciones de Esteban Moctezuma, secretario en turno de la Secretaría de Desarrollo Social.

${ }^{2}$ Cifras anunciadas en la reunión del Banco Mundial y Fondo Monetario Internacional de abril de 2000. 


\section{BIBLIOGRAFÍA}

Aguilar, Genaro. 2000. La pobreza en M éxico ¿Es inevitable? IIEC-UNAM, México.

Aturupane, H., P. Glewwe y P. Isenman. 1994. "Poverty, Human Development and Growth". The A merican Economic R eview. vol. 84, núm. 2, mayo, Papers and proceeding of the Hundred and Sixth Annual Meeting of the American Economic Association, Boston, enero 3-5, 1994, pp. 244-249.

Bancomext. 1998. Exportaciones por entidad federativa. Base de Información.

Banco Mundial. 2000. Informe anual sobre la pobreza en el mundo. Washington.

Bassols Batalla, Ángel. 1972. El N oroeste de M éxico. Un estudio geográfico-económico, UNAM, México.

Bazdrech, C., N isso Bucay, Soledad Loaesa y Nora Lustig. 1992. M éxico. A uge, crisis y ajuste. El Trimestre Económico-FCE, Lecturas, núm. 73, M éxico.

Boltvinik K., Julio y E. Hernández L. 1999. Pobreza y distribución del ingreso en M éxico, Siglo XXI, México.

Camberos C., M. y L. Huesca R. 2001. "Capacidad de consumo y bienestar de los hogares de México y la frontera norte de M éxico". Comercio exterior. vol. 51, núm. 3, marzo. México. Bancomext, pp. 203-210.

CEPAL-ONUDI. 1991. Programa CAN (Análisis de Competitividad de las Naciones). En Progreso técnico y competitividad internacional. Doc. LC/ R 1104, dic.

De la Torre, Rodolfo. 2000. La distribución factorial en el nuevo modelo económico de M éxico. CEPAL, LC/ L.1354.

INEGI. 1992. IX Censo general de población y vivienda 1990. México. 1995. Conteo de Población. México.

1995 y 1996. Cuadernos de información oportuna regional. 20. Semestre. México.

1999. Sistema de Cuentas N acionales, Producto Interno Bruto, 1988, 1993 y 1996. México. 
Kuznets, Simon. 1976. Crecimiento económico moderno. Aguilar. Madrid.

Nafinsa. 1990, 1992 y 1995. La economía mexicana en cifras. México.

Rubli K., Federico y B. Solís M. 1992. M éxico hacia la globalización. Diana. México.

Sen Amartya, K. 1996. "Capacidad y bienestar", en Martha N ussbaum y K. Sen A. (comps.) Calidad de vida, FCE, México.

Sen A martya, K. 1976. "Poverty: A n ordinal approach measur-ment". Econometrica. vol. 44 (2), marzo. Econometric Society, Inglaterra. pp. 219-231.

Smeeding, T. M., M. Higgins y LeeRainwater. 1990. Poverty, inequality and incomedistribution in comparative perspective. The Luxemburg Income Study.

Vega C., Gustavo (coord.). 1991. M éxico ante el Tratado de Libre Comercio con A mérica del $\mathrm{N}$ orte. El Colegio de México-Universidad Tecnológica de México. México.

The World Bank. 2002. W orld Economic Report 2001. Washington, D. C.

2001. Poverty A nnual Report 2000. Washington, D. C.

Wong G., P. 1997. “Estructura de las exportaciones y composición de la economía sonorense", en A. Mungaray y G. García de León, D esarrollo fronterizo y globalización. Unison-ANUIES. México. pp. 19-33. 